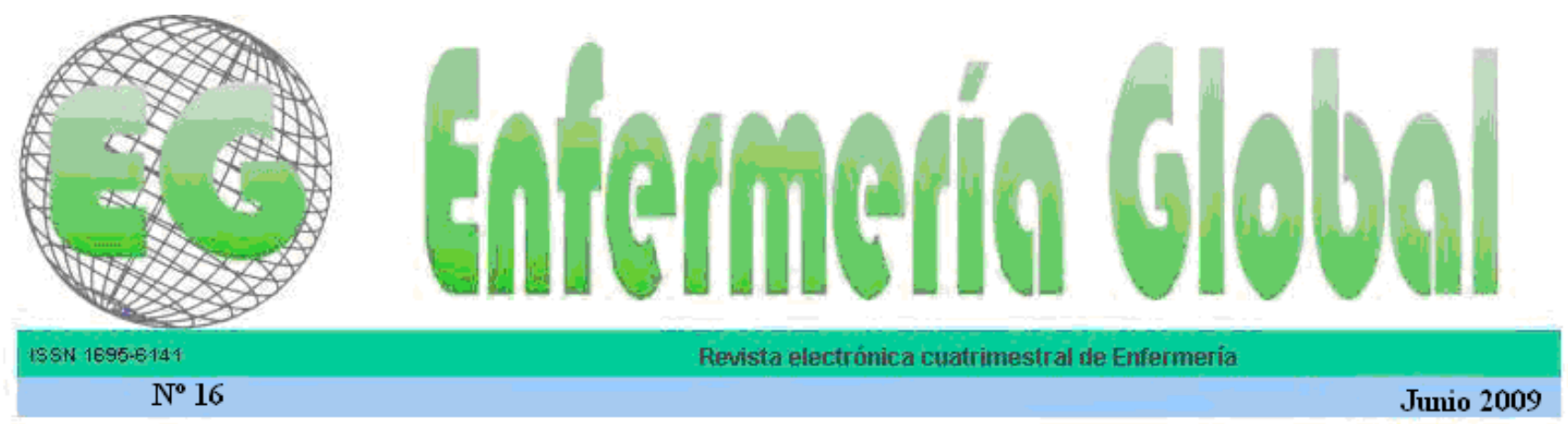

www.um.es/egloball

CLÍNIGA

\title{
EL SABER-HACER DEL ENFERMERO EN EL PROGRAMA DE CONSERVACIÓN AUDITIVA (PCA): DISCUTIENDO UNA PRÁCTICA A PARTIR DEL CONOCIMIENTO DE LOS ESTUDIANTES DE ENFERMERÍA
}

O SABER-FAZER DO ENFERMEIRO NO PROGRAMA DE CONSERVACÃO AUDITIVA (PCA): DISCUTINDO UMA PRÁTICA A PARTIR DO CONHECIMENTO DE GRADUANDOS DE ENFERMAGEM

\author{
${ }^{*}$ Dantas, C. de C., ${ }^{* *}$ Leite, J. L., ${ }^{* * *}$ De Oliveira, M. A., ${ }^{* * * *}$ Dantas, F. de C. \\ *Mestre em Enfermagem pela Escola de Enfermagem Anna Nery/Universidade Federal do Rio \\ de Janeiro EEAN/UFRJ. **Doutora em Enfermagem. Professora titular Emérita UNIRIO. \\ ***Graduando da Escola de Enfermagem do Centro Universitário Celso Lisboa (CEUCEL). \\ *****Graduanda da EEAN/UFRJ. Brasil.
}

Palabras clave: Enfermería, Progrma de Conservación Auditiva, Estudiantes.

Palavras-chave: Enfermagem, Programa de Conservação Auditiva, graduandos

\section{RESUMEN}

Se trata de estudio descriptivo-exploratorio con abordaje cuantitativo. Objetivos: Identificar el conocimiento de los alumnos de último periodo acerca del Programa de Conservación Auditiva (PCA) y analizar este conocimiento, discutiendo sus implicaciones y enfatizando las informaciones necesarias para la actuación del enfermero en el PCA. Los sujetos/escena habían sido estudiantes de enfermería del último periodo de una institución de Río de Janeiro. Los datos se recolectaron en el periodo de marzo a agosto de 2005, a través de un cuestionario semi-estructurado, dado a 43 graduandos. Se ha encontrado $69 \%$ de los sujetos del sexo femenino. Con respecto a la edad, se ha encontrado $27 \%$ con más de 40 años de edad. Trabajan en enfermería $49 \%$ de la muestra y de estos, $37 \%$ en las enfermerías de clínica médica y $10 \%$ con la salud del trabajador. En relación al conocimiento del PCA sólo 7\% respondió conocer el Programa, y las respuestas son incoherentes. Se considera útil que el enfermero, así como el futuro enfermero, tenga consciencia de la importancia del PCA en la preservación y conservación auditiva para el trabajador, porque a través de este, se puede prevenir la integridad auditiva de ellos, mejorando así la calidad de vida y la salud general del trabajador. 
Trata-se de estudo descritivo-exploratório com abordagem quantitativa. Objetivos: identificar o conhecimento de graduandos do último período sobre o Programa de Conservação Auditiva (PCA) e analisar tal conhecimento, discutindo suas implicações e enfatizando informações necessária para atuação do enfermeiro em PCA. Os sujeitos/cenário foram acadêmicos de enfermagem, do último período, de uma instituição particular do Rio de Janeiro. Os dados foram coletados de março a agosto de 2005 no primeiro, através de um questionário semi-estruturado, a 43 graduandos. Encontraram-se $69 \%$ de sujeitos do sexo feminino. Com relação a idade, encontrou-se $27 \%$ com idade superior a 40 anos. Trabalham na enfermagem $49 \%$ da amostra e destes, $37 \%$ em enfermarias de clínica médica e 10\% com a saúde do trabalhador. Em relação ao conhecimento do PCA apenas $7 \%$ responderam conhecer, sendo as respostas, inconsistentes. Considera-se profícuo que 0 enfermeiro bem como o futuro enfermeiro, tenha consciência da importância do PCA na preservação e conservação auditiva para o trabalhador, pois através deste, pode-se prevenir a integridade auditiva dos mesmos, melhorando assim, a qualidade de vida e saúde geral do trabalhador.

\section{ABSTRACT}

It is an exploratory and descriptive study with a qualitative approach. Objectives: To identify the senior nursing students' knowledge of the Hearing Conservation Program (HCP). Additionally to analyze this knowledge and discuss its implications and emphasize the necessary information for nursing performance in the HCP. The subjects were senior nursing students in an institution of Rio de Janeiro, Brazil. The data collection was carried out from March to August 2005 through a semi-structured interview given to 43 graduating nurses. An analysis showed $69 \%$ consisted of females. In regard to age, $27 \%$ were found to be of age 40 or more. Of the sample $49 \%$ work in nursing and of these $37 \%$ work in the nursing rooms of medical clinics and $10 \%$ in worker health. Regarding the knowledge about HCP, only $7 \%$ said they knew about the program and the answers are not coherent. It is of great importance that nurses and future nurses are aware of the importance of HCP in hearing preservation and conservation for the worker, because with this it is possible to ensure their hearing integrity, improving their life quality and general health of the worker.

\section{CONSIDERACIONES INICIALES}

El estudio tiene como objetivo investigar cuestiones relativas a la audición. Este órgano sensorial es una de las estructuras fundamentales que, junto con la comunicación, permite a los seres humanos vivir en armonía en su ambiente social, sea en el trabajo, en el entretenimiento o familiar. Al mismo tiempo, la audición es uno de los sentidos más importantes para el desarrollo psicosocial del individuo y, así, todo lo que se relacione con este sentido merece especial atención ${ }^{(1)}$.

Este estudio debatirá acerca del siguiente objeto de estudio: el conocimiento que los estudiantes tienen acerca del Programa de Conservación Auditiva (PCA). Partiendo de la experiencia previa de los estudiantes de enfermería, en términos de técnica de enfermería, que trabajan en este programa, pero preocupados con la actuación de la enfermería en el ámbito del PCA, se realizó una amplia búsqueda bibliográfica cuyo centro de discusión se basase en cuestiones relacionadas con la conservación auditiva. En lo que respecta a las búsquedas de investigaciones/trabajos que hablasen sobre este tema, realizadas en dos bibliotecas públicas de enfermería de Río de Janeiro y fuentes on-line (Biblioteca Virtual de Salud - BIREME), usando como descriptores los ruidos, la audición, la pérdida de audición y la enfermería, se verificó una cierta escasez de literatura, especialmente en lo que atañe a las investigaciones realizadas por los enfermeros.

En este sentido, se justifica la realización de este estudio en vista de la escasez, así como la posibilidad de construir una investigación que pueda permitir una visión diferente de la 
enfermería en relación con la prevención de la pérdida de la audición, a fin de contribuir a la optimización de la calidad de vida de la salud del trabajador.

En esta cuestión, a partir de la experiencia como técnico de enfermería que trabaja en una gran industria del municipio de Duque de Caxias en el estado de Río de Janeiro, se observó la falta de enfermería en la actuación del PCA. Desde entonces, aumentaron las inquietudes y preocupaciones, acreditándose la importancia y necesidad de una participación activa y dinámica de los profesionales de enfermería en este programa. Estas inquietudes generaron las siguientes preguntas: ¿Cuál es el conocimiento de los estudiantes de enfermería del último período acerca del PCA? ¿Están estos futuros profesionales preparados para la actuación en esta área? ¿Qué informaciones son necesarias para que un profesional pueda desarrollar una atención de calidad? En vista de lo expuesto, se esbozaron los siguientes objetivos:

- Identificar las informaciones o conjunto de informaciones dadas por los estudiantes del último período del curso de graduación acerca del PCA;

- Analizar las informaciones dadas por los estudiantes sobre la actuación del enfermero en este programa;

- Discutir las implicaciones de este conocimiento en la formación del enfermero;

- Verificar la importancia de este cuerpo de conocimiento en la formación del enfermero.

\section{MATERIAL Y MÉTODOS}

A fin de cumplir los objetivos propuestos, se realizó un estudio descriptivo-exploratorio con abordaje cuantitativo. Los sujetos/escenas habían sido estudiantes de dos clases del curso de enfermería, del último periodo de una institución privada, situada en el Estado de Río de Janeiro. La elección por los estudiantes de la graduación reside en el hecho de saber si ellos, los futuros enfermeros, tienen algún conocimiento acerca del PCA, a fin de posibilitar discusiones, entre otros, acerca de la formación de este profesional.

Los datos fueron recolectados en el primer y segundo semestre de 2005, a través de un cuestionario semiestructurado que permitió caracterizar el perfil de los clientes requeridos, así como el conocimiento de ellos acerca del PCA. El cuestionario es una herramienta para la recolección de los datos, constituido por una serie ordenada de preguntas que deben ser contestadas por escrito y sin la presencia del entrevistador ${ }^{(2)}$. Después de haber recolectado los datos, estos fueron sometidos a tratamiento estadístico, que en primer lugar, han conformado una descripción cuantitativa de la sociedad, considerada como un todo organizado. Se define y se delimitan las características de los miembros de esas clases, y después, se mide su importancia o la variación, o cualquier otro atributo cuantificable que pueda contribuir para su mejor comprensión. Pero la estadística puede ser considerada algo más que un medio de descripción racional; es también un método de experimentación y prueba, porque es método de análisis ${ }^{(2)}$.

Para atender los preceptos éticos-jurídicos de la Resolución 196/96, se ofreció a cada participante un término de consentimiento libre y clarificado, después de la explicación de la investigación y dirimiendo las posibles dudas acerca de ella. Se subraya que este estudio fue sometido a la Comisión de Ética de la institución que fue el escenario para la recolección de datos, consiguiendo, por tanto, apoyo jurídico para este estudio. 


\section{PRESENTACIÓN DE LOS RESULTADOS Y DISCUSIÓN}

El cuestionario fue aplicado a dos clases del 9o período del Curso de Graduación en Enfermería de una institución privada. Se distribuyó un cuestionario para cada estudiante en cada clase, con un total de 43. Todos los cuestionarios distribuidos fueron desarrollados, como está explicitado en el Gráfico 1.

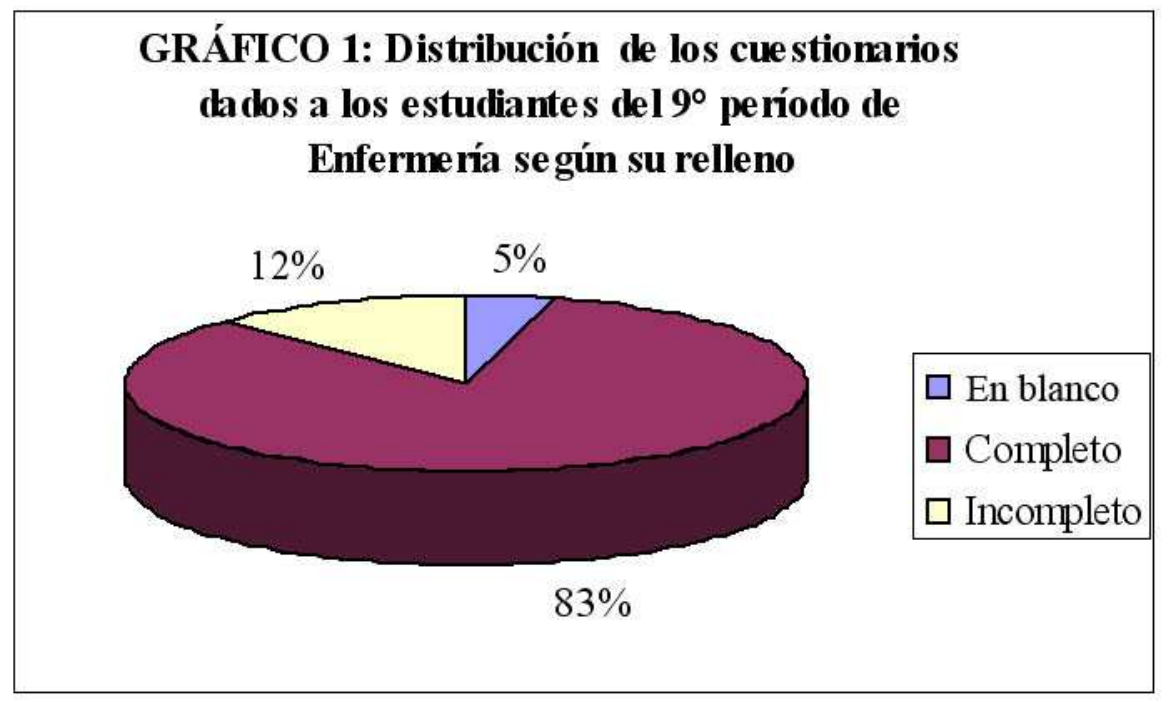

De acuerdo en Gráfico 1 del total de cuestionarios distribuidos, $5 \%$ volvieron en blanco, $12 \%$ incompletos faltando llenar algunas lagunas y $83 \%$ completamente llenos. En cuanto al sexo, se verifica, de acuerdo con el gráfico 2 que la mayoría pertenece al sexo femenino (69\%) y $27 \%$ al sexo masculino.

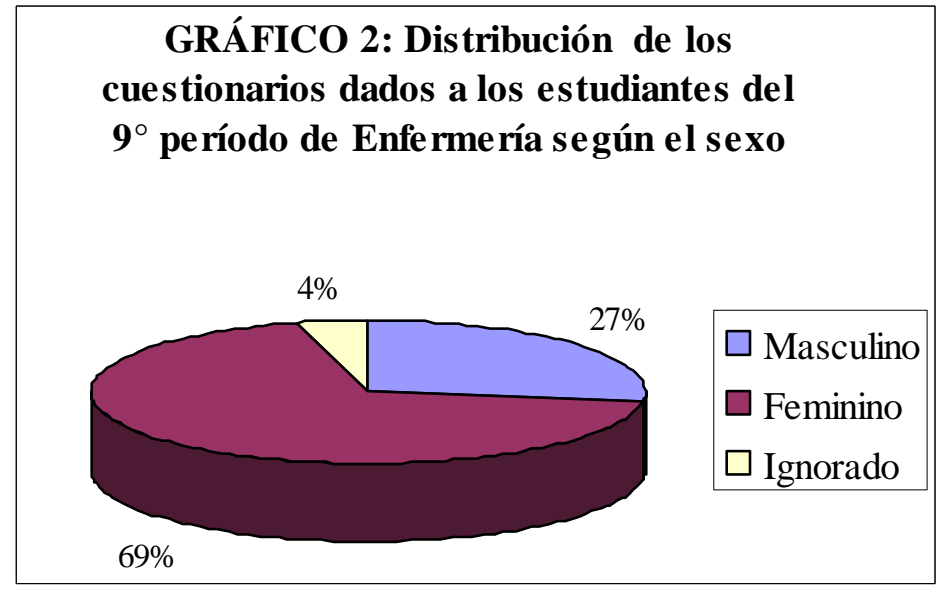

Según el perfil de los clientes investigados de acuerdo con su edad, se observa en el gráfico 3 que la mayoría tiene más de 40 años (27\%), seguido por $26 \%$ de edades comprendidas entre los 26 y 30 años. 


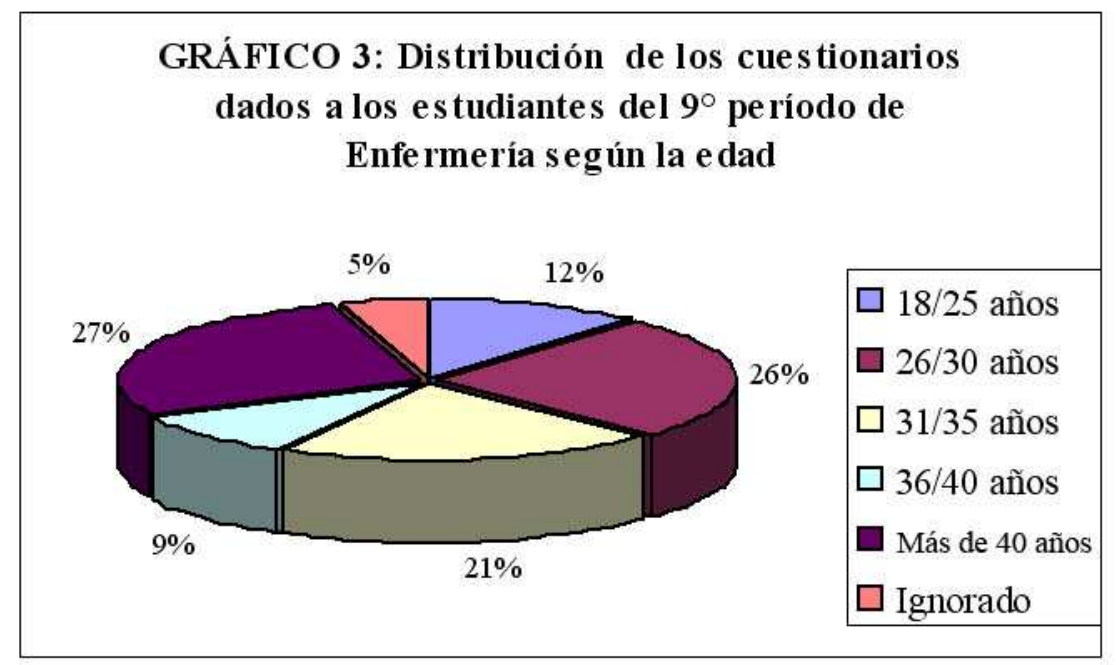

Acerca del desarrollo de actividades laborales en la enfermería, se verificó en el gráfico 4 que de todas las personas investigadas, la mayoría trabaja en esta profesión, totalizando $49 \%$ de la muestra y una cantidad de $26 \%$ que no trabaja.

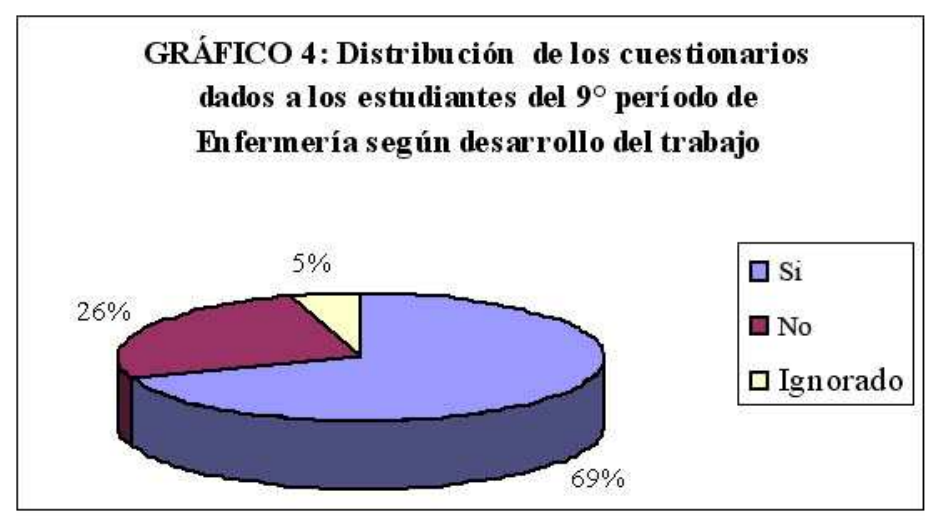

En lo que respecta a los $49 \%$ que realizan actividades en la enfermería, se cuestionó también el sector de actuación. A través del gráfico 5 se observa que la mayoría trabaja en clínica médica (37\%). Énfasis en la salud del trabajador con un total de 10\% trabajando en esta área.

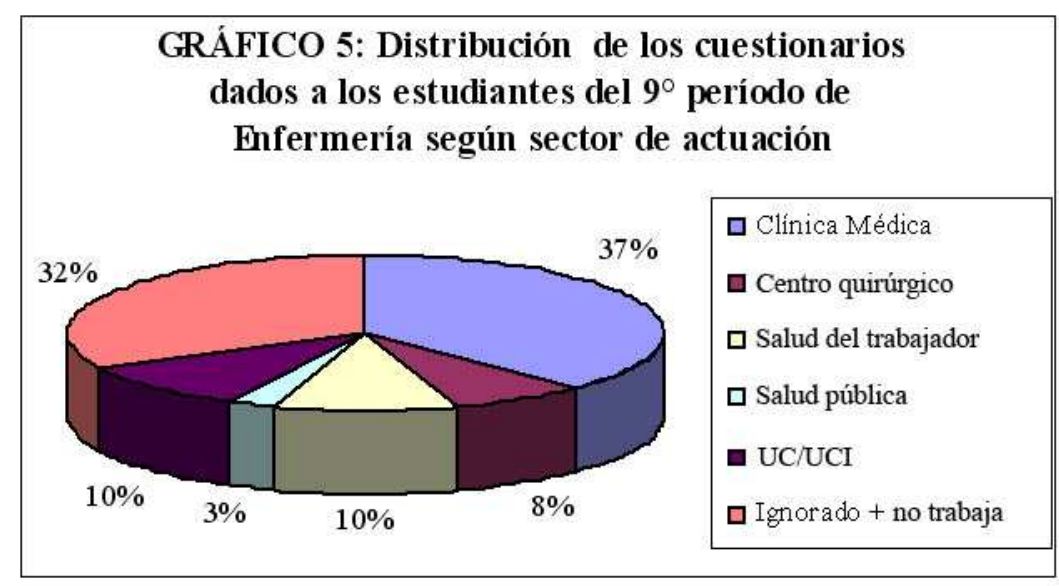


Cuando se les preguntó a los estudiantes acerca del conocimiento sobre el Programa de Conservación Auditiva, se constató que la mayoría no tiene información acerca de este (76\%), seguido de 12\% que sólo ha escuchado sobre el Programa y sólo $7 \%$ conocían el PCA. Estos que tenían alguna información sobre el Programa comprenden el porcentaje que trabaja en el área de la salud del trabajador (Gráfico 6).

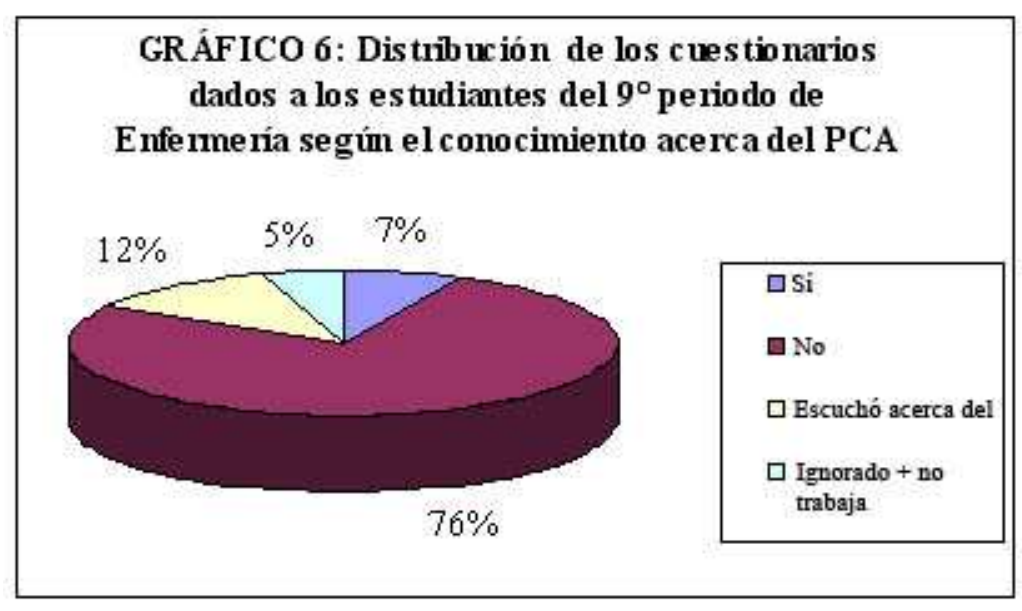

En los datos recolectados, se verificó que la mayoría no conoce el Programa, así estos estudiantes tienen cierta falta de preparación para actuar en este sector. En los ítems del cuestionario relativos a los conocimientos acerca del PCA, a pesar de que 4 sujetos contestaron afirmativamente, un total de $7 \%$, las informaciones descritas por ellos son incompletas y algunas contradictorias. En vista de los escasos conocimientos en esta área del conocimiento, se hace relevante la búsqueda de la literatura para discutir esas cuestiones.

Estos profesionales, recién graduados, si tienen la oportunidad de trabajar en esta área deben buscar ayuda teórica en libros, manuales o Cursos de Post-Grado para hacer valer una asistencia de calidad en el ámbito en que posiblemente actuará. Así, es de suma importancia la participación del enfermero de trabajo en el Programa de Conservación Auditiva dirigido a los trabajadores expuestos a altos niveles de ruido que por medio de las acciones basadas en el proceso de enfermería busca ampliar los conocimientos de esta especialidad y su efectivo papel en el equipo multiprofesional de atención al trabajador ${ }^{(3)}$.

A continuación hablaremos acerca de cuestiones vitales para el conocimiento de los profesionales que tienen afinidad por el tema o que trabajan o desean trabajar en esta área.

\section{SOBRE EL PCA: AYUDAS PARA SU ENTENDIMIENTO}

Algunos autores valoran el PCA como una forma de controlar y reducir la pérdida de la audición. Este resalta factores que pueden conducir a discusia o a acusia, tales como: enfermedades metabólicas, vasculares, isquémicas e iónicas; uso de medicinas, el estrés, la predisposición genética, factores nutricionales, la exposición al ruido y otros ${ }^{(1)}$. Es sabido que la polución sonora, o sea, el ruido en sí, se puede encontrar en diferentes ambientes: en el hospital, el ocio, en las empresas, vías públicas, en nuestras casas, entre otros. En las vías públicas, el ruido que se origina debido al movimiento de las personas, vehículos, maquinaria, puede ser tan perjudicial como el ruido de un ambiente de trabajo ${ }^{(4)}$. Para este último, el ocupacional, deben hacerse esfuerzos en el intento de desarrollar/implantar un 
Programa de Conservación de Auditiva para la prevención de la pérdida de la audición con el fin de controlarla o reducirla.

Según el decreto del INSS (Instituto Nacional de Seguridad Social) con respecto a la pérdida de la audición debido al ruido ocupacional (PAIR) de 1997, esta es una enfermedad de gran prevalencia en nuestro ambiente, que se difunde en numerosos ámbitos de actividad. Esto tiene su principio y predomina, en las frecuencias de $6.000,4.000 \mathrm{y} / 03.000 \mathrm{~Hz}$, avanzando lentamente a las frecuencias de $8.000,2.000,1.000,500$ y $250 \mathrm{~Hz}$, para alcanzar su nivel máximo, en las frecuencias más altas, en los primeros 10 hasta 15 años de constante exposición a altos niveles de presión sonora.

El Programa de Conservación Auditiva es un conjunto de medidas que tiene el objetivo de evitar que determinadas condiciones de trabajo causen deterioro de los umbrales de audición. Debe ser desarrollado dentro de la empresa, a través de un equipo interdisciplinario, teniendo al enfermero como uno de los profesionales de gran importancia para el desarrollo e implantación de este programa, además de médicos e ingenieros, especialmente ${ }^{(5)}$.

Dada la preocupación por la salud de los trabajadores, el Ministerio del Trabajo defiende Normas Reguladoras (NR) a fin de preservar la salud del profesional durante el desarrollo de actividades laborales. El enfermero debe tener tales nociones, ya que el ruido puede estar presente en cualquier ambiente. Por lo tanto, este profesional debe subvencionar en parámetros y programas destinados a evitar los riesgos a todos en su ambiente de trabajo, ofreciendo mejores condiciones para todos.

Es importante que el enfermero tenga conocimiento que la NR-9 del Decreto $\mathrm{n} \cong 3.214$ del Ministerio del Trabajo ${ }^{(6)}$, que establece que todas las empresas deben tener un Programa para Prevenir los Riesgos Ambientales. Cuando se tiene el nivel de presión sonora alto como uno de los elementos de riesgo señalados por este programa, la empresa debe organizar bajo su responsabilidad, un Programa de Conservación Auditiva. Las informaciones siguientes se refieren al desarrollo de un PCA, que se basa en la literatura afín, las normas y reglamentos $(1,4,6,7,8,9,10,11,12)$, que atañen a las recomendaciones mínimas para el desarrollo de un Programa de Conservación Auditiva. Así, para tener la eficacia de este programa, el equipo que participa en este proceso, especialmente en lo que respecta al enfermero, debe buscar las siguientes etapas:

\section{1) Monitoramiento de la exposición a altos niveles de presión sonora:}

En primer lugar deberá hacerse una evaluación del sitio con respecto al ruido. Por lo tanto, se debe hacer una evaluación minuciosa de los niveles de presión sonora elevados de la empresa por sector con objetivo de:

- evaluar la exposición de los trabajadores a los riesgos;

- determinar si los altos niveles de presión sonora presentes pueden interferir en la comunicación y la percepción audible de las señales de advertencia;

- priorizar los esfuerzos de control del nivel de presión sonora elevado, definir y establecer prácticas para proteger a la audición;

- identificar a los trabajadores que participarán en el PCA;

- evaluar el trabajo de control del nivel de presión sonora elevado. 


\section{2) Controles de ingeniería y administrativos:}

Los controles de ingeniería y administrativos son los elementos más importantes de un PCA, ya que sólo a través de la reducción del nivel de presión sonora elevado o de la exposición, se puede prevenir el daño causado por el nivel de presión acústica elevado. Por lo tanto, los profesionales de la ingeniería, en particular, tienen un papel muy importante en esta etapa, ya que trabajan con cuestiones que se pueden definir como todo cambio o sustitución de equipos que provoquen cambio físico en el origen o en la transmisión del nivel de presión sonora elevado (a excepción de los EPIs), reduciendo los niveles sonoros que llegan al oído del empleado. Otro grupo de profesionales es el de carácter administrativo, que puede contribuir para hacer más flexible el horario de trabajo y operaciones de las máquinas, buscando reducir la exposición de los funcionarios, así como el turno de los empleados en las áreas de alto nivel de presión sonora, funcionamiento de ciertas máquinas en turnos $u$ horarios con menos personas presentes, entre otros.

\section{3) Monitoramiento de audiometría:}

Cabe a los profesionales de la salud, especialmente a los enfermeros, hacer la gestión de este proceso junto a otros profesionales de la salud involucrados en este (los fonoaudiólogos y los médicos), dar prioridad para esta etapa en la identificación y posterior mantenimiento y control de posibles cambios auditivos. Esto sirve como un parámetro de la eficiencia y eficacia de la aplicación del PCA. Conforme el Boletín 03, son objetivos del monitoramiento audiométrico:

- establecer la audiometría inicial de todos los trabajadores;

- identificar el estado auditivo (audiogramas normales y alterados), haciendo un acompañamiento periódico;

- identificar las personas con necesidad de acudir al médico otorrinolaringólogo para comprobar posibles cambios en el oído medio;

- advertir a los trabajadores acerca de los efectos del nivel de presión sonora, ofreciéndoles los resultados de cada examen;

- contribuir de manera significativa a la implantación y la eficacia del PCA;

- Ios audiogramas iniciales deben ser utilizados como una referencia y comparados, individual o colectivamente, con los exámenes realizados posteriormente, para verificar si las medidas de control del nivel de presión sonora elevado están siendo eficaces;

- el diagnóstico de la pérdida de la audición no desclasifica al trabajador en el desempeño de sus funciones laborales. La vigilancia debe ser utilizada como prevención de la progresión de la pérdida de la audición inducida por el ruido y no como un medio de excluir a los trabajadores de sus actividades;

- Los trabajadores deben recibir una copia de los resultados de sus audiogramas.

\section{4) Indicación del Equipo de Protección Individual - EPI:}

Una vez en un área de riesgo para la audición algunas medidas deben ser tomadas con respecto a la protección individual. El protector auricular tiene el objetivo de atenuar la potencia de la energía sonora transmitida al aparato auditivo. 
Según el Boletín, la selección del EPI más adecuado para cada situación es responsabilidad del equipo de ejecución del PCA. Así, algunos aspectos deben ser considerados en cuanto a la selección de ellos:

- nivel de atenuación que represente una efectiva reducción de energía sonora que llega a las estructuras de la cóclea;

- modelo que se ajuste a la función realizada por el trabajador;

- confort;

- aceptación de la protección por el trabajador.

\section{5) Educación y motivación:}

Para que todo este proceso sea efectivo, se debe buscar la adhesión de todas las personas que comparten el mismo ambiente. Estas deben incluirse en los programas de aclaración acerca del tema, realización de las conferencias con el objetivo de hacer el empleado tomar conciencia de la importancia de la prevención y, principalmente, para motivar a todos, ofreciendo una infraestructura adecuada y flexibilizando los aspectos administrativos y operativos. Esta sistematización tiene el objetivo de prevenir la pérdida de la audición. Se recomienda que los trabajadores comprendan al menos las siguientes cuestiones:

- los efectos a la salud causados por la exposición a niveles de presión sonora elevados;

- la interpretación de los resultados de los exámenes audiométricos;

- la concepción, la metodología, la estrategia y la interpretación de los resultados de las evaluaciones ambientales;

- medidas de protección individual y colectiva posibles.

\section{6) Mantenimiento de registros:}

Dada la importancia del registro para el adelanto científico, se deberá primar el registro y consecutivo archivo de todos los datos recolectados en el proceso de desarrollo y mantenimiento del PCA. El enfermero debe cuidar que la persona jurídica archive todos los datos relacionados con los resultados de la audiometría, así como las evaluaciones ambientales y las medidas adoptadas de protección colectiva, incluido disponer a los empleados y otros interesados como los órganos de fiscalización, dada la importancia de la participación de todos para lograr el éxito en este proceso.

\section{7) Evaluación de la eficacia y la eficiencia del programa:}

Para mejorar todo el proceso de desarrollo y mantenimiento del PCA se deberán realizar procesos de evaluación periódicos con el fin de mejorar cuanto sea necesario, haciendo sus ajustes. El pleno alcance de los objetivos se establece a través de evaluación sistemática y constante. El uso del check-list para supervisar la aplicación del PCA puede ser muy útil y facilitador de la evaluación, debiéndo prestar atención a los siguientes aspectos básicos:

- la evaluación de la perfección y la calidad de los componentes del Programa;

- la evaluación los datos del examen audiológico;

- opiniones de los trabajadores. 
Estos son las etapas recomendadas para el desarrollo y mantenimiento de un PCA. El enfermero debe buscar la mejora en esta área de actividad para ofrecer una mejor asistencia a las personas que desarrollan trabajos en lugares donde el ruido es un factor que puede conducir a graves complicaciones futuras. Unido a esto, esta el hecho de ser el enfermero uno de los profesionales con gran potencial para, junto al equipo interdisciplinar, desarrollar un trabajo de calidad en la salud del trabajador.

\section{CONSIDERACIONES FINALES}

El perfil de la muestra de este estudio se caracterizó mayoritariamente como femenino, total de $69 \%$ y sólo $27 \%$ del sexo masculino. Con respecto a la edad, encontramos $27 \%$ de más de 40 años, seguido por $26 \%$ de edades comprendidas entre los 26-30 años. En lo que respecta al desempeño de actividades laborales, 49\% trabajan en enfermería como un profesional de nivel medio, y de estos, 37\% trabajan en la enfermería de clínica médica y una cantidad de $10 \%$ en el área de la Salud del trabajador.

Con respecto a los conocimientos de estos futuros profesionales acerca del PCA, se constató que en las dos clases del último periodo del curso de graduación en enfermería, $76 \%$ no conocen el Programa de Conservación Auditiva, seguido por 12\% que sólo escuchó acerca del Programa y sólo 7\% había dicho que conocían el programa. Sin embargo, cuando solicitado, a través del instrumento, que contestasen preguntas específicas acerca de este programa, casi ninguno supo describir el programa de modo correcto.

En lo que respecta a la formación de estos profesionales, existe una laguna en este sector de actividad. Se constató, al observar los resultados de la investigación en los sujetos del estudio, que durante la graduación casi nada se ha trabajado acerca de la salud del trabajador y que, en particular, el PCA no fue discutido en las actividades realizadas en el aula o fuera de ella. Algunos dijeron conocer alguna información sobre el grupo que está trabajando en la salud del trabajador y, por tanto, algo han trabajado en esta dirección, pero sin la profundidad técnico-científica necesaria.

Así se hace necesaria ayuda para la institución y otras que quizás no aborden la salud del trabajador en el currículo, repensar este campo de actuación de la enfermería e insertarllo en el proceso de enseñanza aprendizaje, con vistas al campo de actuación de la enfermería, ya que incluso, a partir de 2003, con la Resolución COFEN-286 ${ }^{(13)}$, el enfermero puede preparar, emitir y firmar el informe técnico sobre las condiciones ambientales de trabajo, estando el enfermero obligado a mantener registros sistemáticos en el prontuario del empleado.

El Programa de Conservación Auditiva es un conjunto de medidas que tiene como principal objetivo la protección de la Salud del Trabajador para impedir que los trabajadores expuestos a niveles peligrosamente altos de ruido desarrollen pérdida de la audición inducida por el ruido ocupacional.

Es útil que el enfermero y el futuro enfermero, tenga consciencia de la importancia del PCA en la preservación y conservación auditiva para el trabajador, porque a través de este, se puede preservar la integridad de su audición. Junto a esto y a través de la gradual transformación de la organización del trabajo, de las condiciones de trabajo y de los procesos de trabajo, intentamos rescatar el sentido mayor del trabajo, o sea, una actividad sin sufrimiento, dolor, enfermedad o muerte, mejorando así la calidad de vida y la salud en general. 
Por lo tanto, las informaciones trabajadas aquí, acerca de los programas de conservación auditiva, en ningún momento tuvieron la pretensión de agotar el tema, debido a su alta complejidad. Requiere aún, la aplicación del cuestionario en otras instituciones, ya que esto es algo desconocido localmente y no fuera del ámbito de aplicación de los currículos de la graduación en enfermería.

Se espera que algunos parámetros trabajados en este estudio hagan posibles las discusiones/reflexiones en el ámbito de la enseñanza, investigación y de la asistencia acerca de la formación y actuación del enfermero en el área de la salud de los trabajadores como un todo.

\section{REFERENCIAS}

1. Siviero AB, Fernandes MJ, Lima JAC, Santoni CB, Bernardi APA. Prevalência de perda auditiva em motoristas de ônibus do transporte coletivo da cidade de Maringá - PR. Rev CEFAC 2005 julho-outubro; 7(3): 376-81 2005.

2. Lakatos EM, Marconi MA. Metodologia do trabalho científico. São Paulo: Atlas; 2001.

3. Baggio MCF, Marziale MHP. A participação da enfermeira do trabalho no programa de conservação auditiva. Rev. Latino-am Enfermagem 2001setembro-outubro; 9(5):97-9.

4. Bernardi APA, Saldanha Junior OM. Construindo o programa de conservação auditiva (PCA). In: Bernardi APA organizador. Audiologia ocupacional. São José dos Campos: Pulso; 2003. p. 49-65.

5. Norma Regulamentadora $n^{\circ} 9$ - NR9. Programa de Co ntrole Médico de Saúde Ocupacional. Diário Oficial da União 1994; 30 dez.

6. Comitê Nacional de Ruído e Conservação Auditiva. Boletim no 6. Recomendações mínimas para a elaboração de um PCA. São Paulo: Comitê Nacional de Ruído e Conservação Auditiva; 1999.

7. Comitê Nacional de Ruído e Conservação Auditiva. Boletim n¹. Perda Auditiva Induzida pelo Ruído Relacionado ao Trabalho. São Paulo: Comitê Nacional de Ruído e Conservação Auditiva; 1994.

8. Gabas GC. Programa de Conservação Auditiva - Guia Prático 3M. 2004. [on-line]. Disponível em: http://www.3m.com/intl/br/saude ocupacional seg ambiental/pdf/pca final.pdf. Acesso em: 24 maio 2006.

9. Norma Regulamentadora no 7 - NR7. Programa de Controle Médico de Saúde Ocupacional. Diário Oficial da União 1994; 30 dez

10. Comitê Nacional de Ruído e Conservação Auditiva. Boletim n³. Condutas na Perda Auditiva Induzida pelo Ruído. Belo horizonte: Comitê Nacional de Ruído e Conservação Auditiva; 1995.

11. Norma Regulamentadora $n^{\circ} 15$ - NR15. Atividades e operações insalubres, CLT Capítulo V, Título II. Diário Oficial da União 1978; 8 jun.

12. Farias FF, Dantas AA. A Perda Auditiva Induzida Pelo Ruído (PAIR) nos músicos de Aracaju 2001; 254(22):46-57.

13. Conselho Federal de Enfermagem - COFEN. Resolução COFEN - no 286/2003. Dispõe sobre a autorização para o enfermeiro do trabalho elaborar, emitir e assinar laudo técnico de condições ambientais de trabalho previsto no perfil profissiográfico previdenciário PPP. 